\title{
Advances in surgical treatment of lymphedema
}

\author{
Maureen Beederman, David W. Chang \\ Section of Plastic and Reconstructive Surgery, The University of Chicago, Chicago, IL, USA
}

An estimated 250 million people worldwide suffer from lymphedema. In the past, the firstline option for treatment was nonsurgical management, either in the form of compression garments or wrapping, or comprehensive decongestive therapy, with debulking surgery reserved for the more advanced cases. However, with improvements in microsurgical techniques and imaging modalities, surgical intervention is increasingly being utilized. This review highlights recent advancements in the surgical treatment of lymphedema, specifically focusing on improvements in imaging, surgical techniques, and prevention of lymphedema.

Keywords Lymphedema / Surgery / Microsurgery / Advances / Treatment

\author{
Correspondence: David W. Chang \\ Section of Plastic and Reconstructive \\ Surgery, The University of Chicago \\ Medicine \&t Biological Sciences, 5841 \\ S. Maryland Ave., Rm J641, MC 6035, \\ Chicago, IL 60637, USA \\ Tel: +1-773-702-6302 \\ Fax: +1-773-702-1634 \\ E-mail: \\ dchang@surgery.bsd.uchicago.edu
}

\section{INTRODUCTION}

Lymphedema is a chronic, often progressive, condition caused by insufficient lymphatic drainage and the subsequent buildup of protein-rich interstitial fluid. This causes significant limb swelling, inflammation, and at later stages, fibrosis. Lymphedema can affect both upper and lower extremities, and can be classified as either primary, where there is an intrinsic problem with the lymphatics such as agenesis or dysplasia, or secondary, where normal lymphatic vessels and drainage pathways are disrupted or damaged. In the developed world, the leading cause of lymphedema is following oncologic treatments, including lymphadenectomy and/or radiation, of the axilla or groin, commonly following breast cancer, gynecologic or urologic cancers, or melanoma. As oncologic treatments continue to improve, so too does life expectancy, and its associated morbidities, of which lymphedema is one. Patients with lymphedema often experience significant pain and discomfort, limb heaviness, frequent bouts of cellulitis, and an overall decreased quality of life [1].

Although surgical treatment of lymphedema dates back to the early 1900s with Charles' description of his debulking technique for scrotal and lower extremity lymphedema [2], advancements in microsurgical techniques have given surgeons the ability to address the physiologic aspects of lymphedema with vascularized lymph node transplants (VLNT) and lymphovenous bypass (LVB). The goal of this review is to provide an overview of recent advancements in the care of lymphedema patients, focusing on improvements in imaging, surgical techniques, and lymphedema prevention.

\section{COMBINED SURGERIES}

While there have been many studies documenting the efficacy of both VLNT and LVB separately for the treatment of lymphedema $[3,4]$, there is a paucity of data on the combined simultaneous use of both VLNT and LVB. Several recently accepted and published studies from our group describe our success using this combined technique on 220 patients during a 5.5-year period. Patients showed significant improvements in both volume reduction and Lymphedema Life Impact Scale (LLIS) scores at all time points postoperatively (Figs. 1-4) [5]. Furthermore, we showed that both patients with upper and lower ex- 

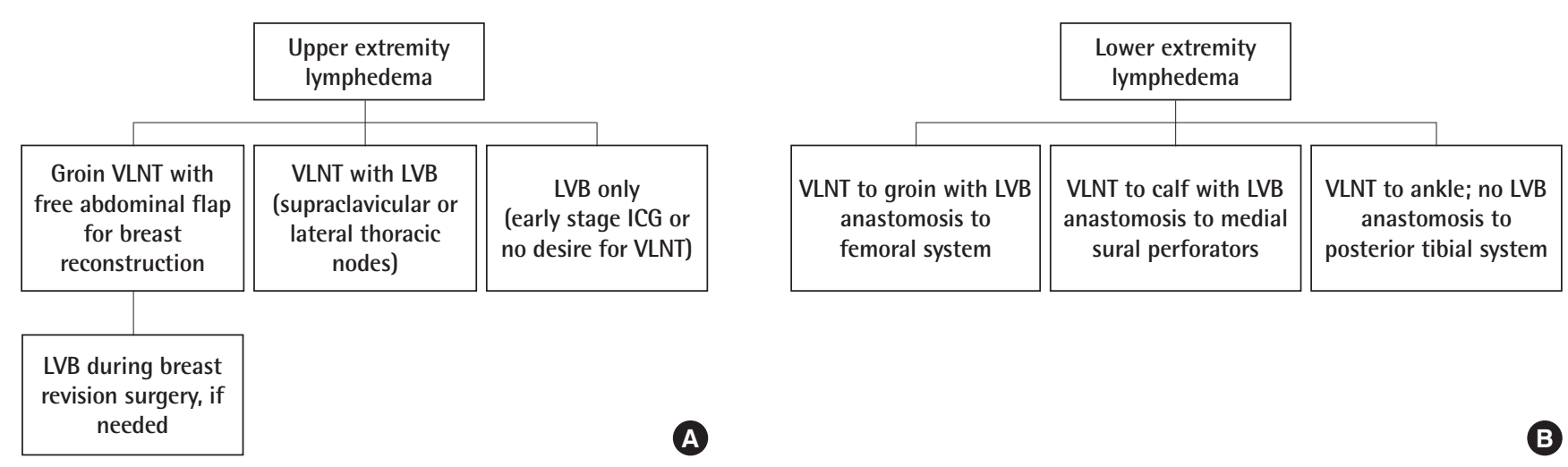

Fig. 1. Algorithm for patient selection for upper extremity lymphedema (A) and lower extremity lymphedema (B). The decision of what surgical approach to use is made based on preoperative discussions with the patient, as well as indocyanine green lymphography (ICG) findings intraoperatively. Early-stage lymphedema is often amenable to lymphovenous bypass (LVB) alone, and is a good option for patients wanting to have minimal surgery. For patients with upper extremity lymphedema (A) who are undergoing simultaneous abdominal flap-based breast reconstruction, groin vascularized lymph node transplant (VLNT) is typically performed with the breast flap in one procedure. If needed, LVB is performed in a delayed fashion with subsequent breast revisions. For the majority of patients, a combined approach of VLNT and LVB is preferred, using either the supraclavicular or lateral thoracic lymph nodes. For lower extremity lymphedema (B), the decision regarding VLNT placement depends on the presence of existing surgical scars, as well as ICG findings and whether LVB can be performed distally in the leg. If no LVB sites are found, the VLNT are placed distally in the ankle. Reprinted from Beederman et al. Ann Surg 2020 Sep 1 [Epub]. https://doi.org/10.1097/ SLA.0000000000004457, with permission from Wolters Kluwer Health, Inc. [5].

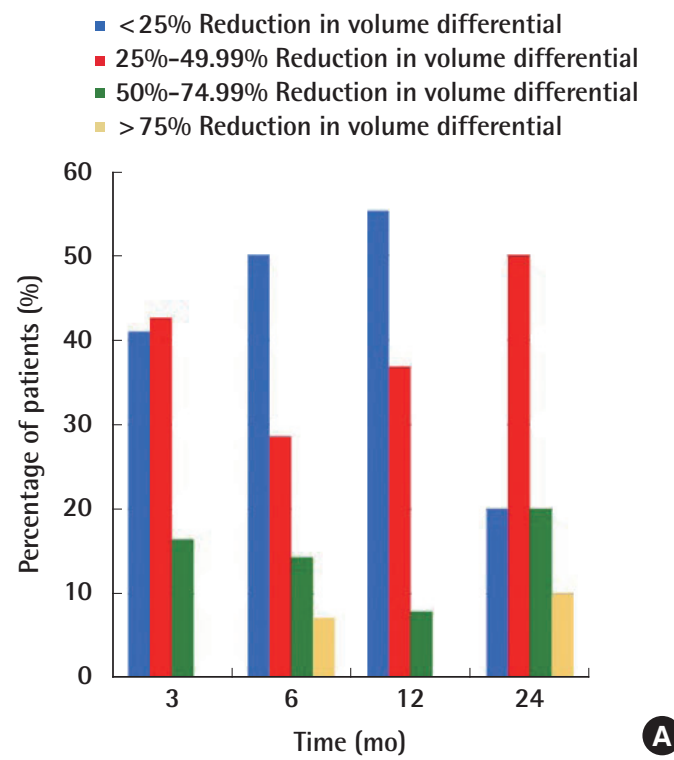

- $<25 \%$ Reduction in volume differential

- 25\%-49.99\% Reduction in volume differential

- 50\%-74.99\% Reduction in volume differential

A

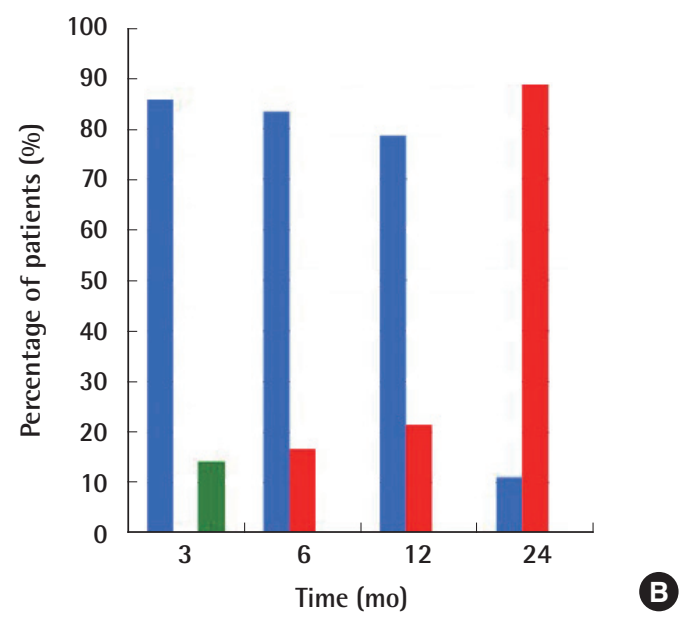

Fig. 2. Change in volume differential over time. Graphical representation of range of improvement in volume differential between affected and unaffected upper (A) and lower (B) extremities at early (3 and 6 months) and late (12 and 24 months) time points. (A) Close to 80\% of upper extremity patients had either a $<25 \%$ (blue) or 25\%-49.99\% (red) improvement at 3 and 6 months. At 24 months, the majority of upper extremity patients (50\%) had a 25\%-49.99\% (blue) reduction in volume differential between their limbs. (B) For lower extremity patients, the majority who improved exhibited a modest reduction in volume differential $(<25 \%$, blue), during the $3-, 6-$, and 12 -month time points. A greater reduction in volume differential was seen at 24 months, with over 80\% of patients who improved showing a 25\%-49.99\% reduction (red). It is likely that more patients tended to show greater increase in volume reduction at the later time points due to optimized functioning of VLNT by that time. Reprinted from Beederman et al. Ann Surg 2020 Sep 1 [Epub]. https://doi.org/10.1097/SLA.0000000000004457, with permission from Wolters Kluwer Health, Inc. [5].

tremity, as well as primary and secondary lymphedema, may benefit from this combined approach. When possible, we favor performing VLNT and LVB together, as these two surgical interventions work by different mechanisms. The impact of LVB can often be felt almost immediately by patients, as excess lymphatic fluid is diverted into the venous system. It is likely that early postoperative limb improvements are mostly the result of LVB. VLNT, on the other hand, is likely responsible for the de- 

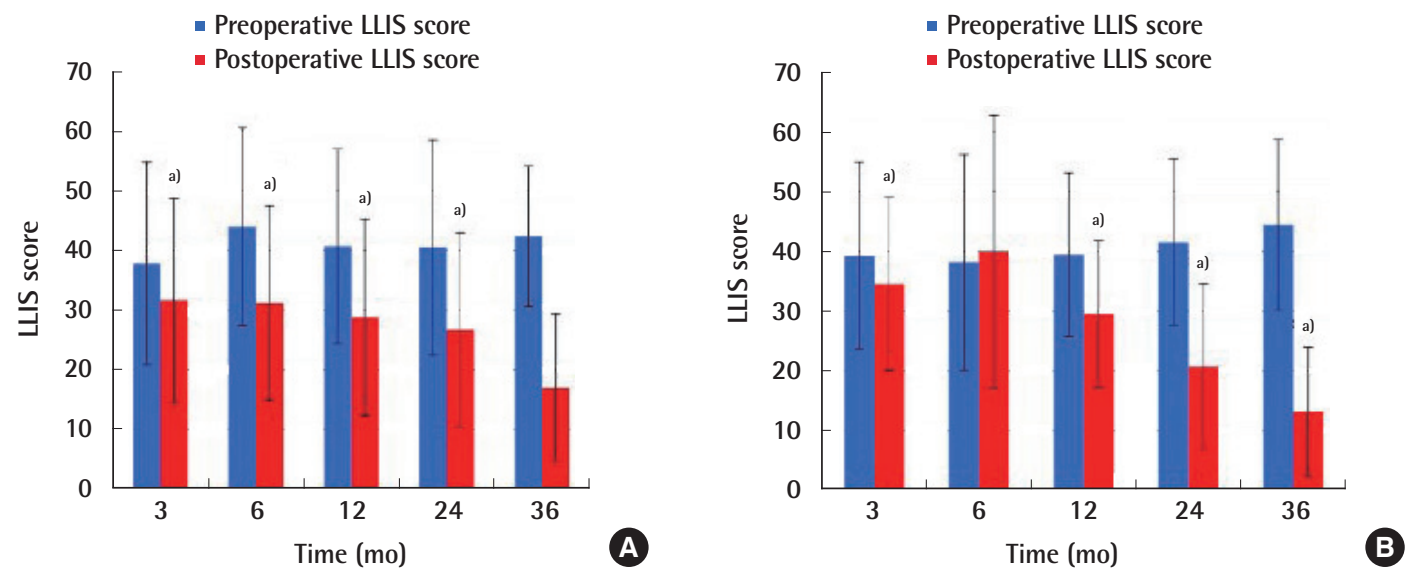

Fig. 3. Change in Lymphedema Life Impact Scale (LLIS) scores over time. LLIS scores down-trended at each time point postoperatively for secondary upper extremity patients (A), compared to preoperative scores. This was also true for secondary lower extremity (B), except for 6-month follow-up, where data is not statistically significant. $\left.{ }^{a}\right)$ This decrease was statistically significant $(\mathrm{P}<0.05)$ at all other time points postoperatively, with the exception of 36 months for upper extremity patients, which is likely due to low numbers of patient data at this time point. The greatest improvement was seen at 36-month postoperative for both upper and lower extremities, with LLIS scores just over 10, compared to >40 preoperatively. Reprinted from Beederman et al. Ann Surg 2020 Sep 1 [Epub]. https://doi.org/10.1097/SLA.0000000000004457, with permission from Wolters Kluwer Health, Inc. [5].
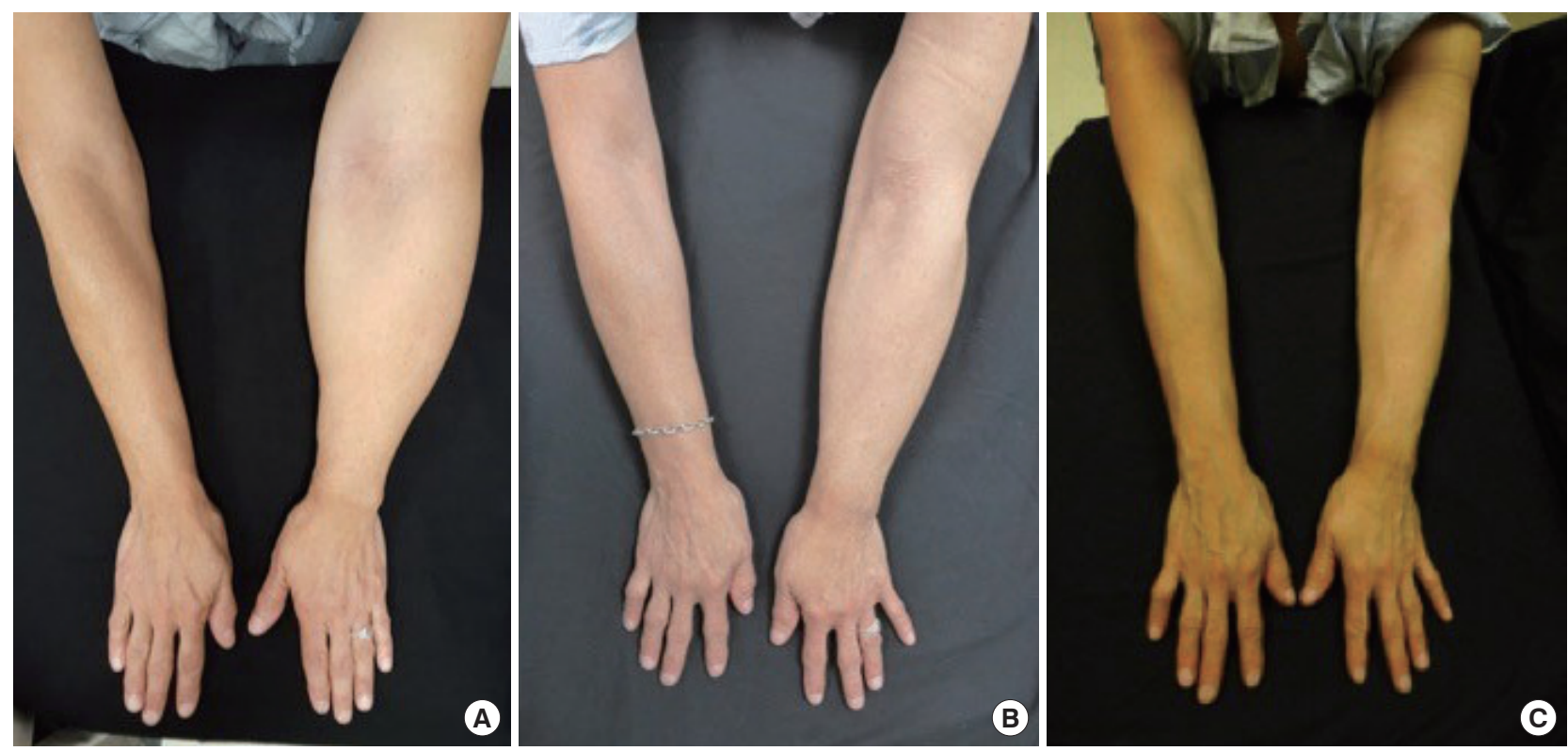

Fig. 4. A 63-year-old woman with breast cancer-related left arm lymphedema. (A) Preoperatively, her left arm was 54.5\% larger than her right arm. Her Lymphedema Life Impact Scale (LLIS) score was 41. (B) At 2-year postoperative follow-up, she had 47.9\% reduction in volume differential and her LLIS improved to 3. (C) At 3-year postoperative follow-up she had 69.2\% reduction in volume differential and her LLIS improved to 1. Reprinted from Beederman et al. Ann Surg 2020 Sep 1 [Epub]. https://doi.org/10.1097/SLA.0000000000004457, with permission from Wolters Kluwer Health, Inc. [5].

layed, sustained improvements seen more than 2 years postoperatively, as its mechanism of action requires more time for optimal functioning. Similar findings have been subsequently published by others [6].

Because excess limb volume is comprised of both fluid and fibrofatty components, the combined use of both physiologic and debulking techniques is also increasingly being utilized. In their retrospective study, Brazio and Nguyen [7] describe their use of physiologic surgeries (either VLNT or LVB), combined with debulking procedures (liposuction). The authors describe their treatment algorithm, in which the decision regarding whether physiologic or debulking surgery should be performed first, or whether they should be performed simultaneously, is based on the severity/stage of lymphedema, as well as whether their 
swelling is predominantly fluid or fibrofatty in nature. They show that all patients experienced a decrease in limb volume, as well as reduced daily compression usage and reduced incidence of cellulitis [7].

Given the lack of uniformity in the many published studies on surgical treatment of lymphedema, it can be difficult to draw conclusions regarding which treatments and combinations of treatments are optimal. To this end, recently published guidelines from a consensus conference of lymphedema experts held in 2017, during which a meta-analysis of clinical trials was performed, provide some guidance [8]. The authors conclude that there is evidence that both VLNT and LVB are effective in reducing limb volume in patients with lymphedema, although there is currently no definitive answer on which technique is more effective. They also note that liposuction and compression is an effective technique in the treatment of lymphedema, and that liposuction may be combined with physiologic procedure, although the best timing of this is unknown.

As physiologic surgical interventions become more popular and widespread, there is an increasing amount of information on how an individualized surgical approach, based on a patient's history, physical exam, imaging results, may lead to improved outcomes. There are a number of treatment algorithms in the literature. In their recent paper, Kwon et al. [9] outline their approach to treatment of breast cancer-related lymphedema, with a combination of conservative nonoperative treatments, LVB based on ultrasound and indocyanine green lymphography (ICG) lymphography imaging, axillary scar release, and VLNT. Their algorithm is based on the presence of scarring and loss of soft tissue in the axilla. Similarly, Schaverien and Coroneos [10] detail their approach to patients with lymphedema through the use of several different algorithms. These algorithms can be used to help guide decision-making regarding appropriate selection of anastomotic techniques for LVB based on the sizes of the lymphatic and superficial venules, donor site choices for VLNT and subsequent recipient site placement, and overall treatment options for patients depending on the severity of their lymphedema.

\section{IMAGING TECHNIQUES}

Improvements and advances in imaging techniques have impacted not only the diagnosis of lymphedema, but also the treatment options available to patients. Lymphoscintigraphy, which has long been considered the gold standard in the diagnosis and evaluation of lymphedema, involves the subdermal injection of radiolabeled tracer distally in the extremity in question. The tracer is then is taken up by the lymphatics and travels proximal- ly to reach the nodal basin. In patients with lymphedema, this transport is often impaired, leading to delayed uptake and dermal diffusion. While lymphoscintigraphy is helpful in detecting the presence of lymphedema and assessing the function of lymphatic channels, there are several drawbacks to this modality, including poor resolution and a lack of information on the lymphatic anatomy and accompanying vasculature.

In addition to lymphoscintigraphy, ICG has become a popular imaging technique, especially for preoperative or intraoperative imaging of lymphatic channels. ICG is injected intradermally in the distal extremity, where it binds to albumin and is taken up by surrounding lymphatic channels. This provides information on where channels are located, as well as areas of blockage and dermal backflow. ICG is useful in the detection of superficial lymphatics and therefore, aids in the decision-making process regarding where to perform LVB. Although useful, ICG is limited in that it can only detect superficial lymphatic channels within $1.5-2 \mathrm{~cm}$ of the skin surface, may be unable to detect lymphatic channels amidst dermal backflow patterns, and also provides no information about the surrounding vascular anatomy.

Imaging using ultrasound has also been successfully used to help identify potential LVB targets in patients with lower extremity lymphedema. In 2015, Hayashi et al. [11] published their study on the feasibility of using ultrasound to locate lymphatic vessels, comparing the location of lymphatics detected with ICG lymphography to that detected with ultrasound. Although this study was performed in healthy volunteers without lymphedema, it concluded that ultrasound was highly sensitive (95.5\%) and specific (92.9\%) in detecting superficial lymphatics. Other studies have since been published on the effectiveness of ultrasound in detecting lymphatic vessels in lymphedematous limbs, showing that use of ultrasound resulted in detection of larger lymphatics, reduced operative time, and improved volume reduction postoperatively, when compared to ICG lymphography [12]. Recently, the use of ultra-high-frequency ultrasound imaging has also shown promise, resulting in the detection of smaller lymphatics with improved resolution [13].

Newer imaging techniques, including magnetic resonance angiography (MRA), have provided increasingly detailed information for providers. For example, MRA not only enables us to diagnose lymphedema, it also gives information regarding the distribution of fluid and/or fibrofatty tissue in the limb, as well as information regarding vascular status and possible venous stenosis. A recent study by Dayan et al. [14] shows the impact that MRA can have on the treatment of lymphedema patients. In this study, they examine the MRA results in patients with either primary or secondary lower extremity lymphedema, and subjectively classify and grade the fluid or fat distribution in the 
limb. They make the important observation that even patients within the same International Society of Lymphology (ISL) class may have different percentages of fat and fluid, which can impact treatment options and results. In another study, Dayan et al. [15] also show the utility of preoperative MRA in assessing the anatomy of donor groin lymph nodes prior to VLNT, an appropriately understanding of which is crucial to avoid iatrogenic donor site lymphedema.

In addition to determining the composition of a lymphedematous limb, magnetic resonance lymphangiography (MRL) is also useful in determining precise incision placement in which to perform LVB. Yasunaga et al. [16] examined the ability of preoperative MRL to locate usable lymphatic vessels for LVB, and found that this technique had a precision of $94 \%$, with both a high positive predictive value and a high anastomotic rate for LVB. The results from this study are also supported by the findings from a recent systematic review by Forte et al. [17], which looked at the impact of MRL on preoperative planning for physiologic lymphedema surgery. Although limited to include only nine studies, the analysis concluded that MRL potentially improves surgical outcomes with additional studies showing that this imaging technique is more sensitive than others (lymphoscintigraphy, ICG, and ultrasound) in detecting lymphatic abnormalities.

\section{TREATMENTS OF ADVANCED STAGE LYMPHEDEMA}

While there has been much research on the effects and improvements in volume reduction with physiologic surgical techniques, many of these studies are limited in that they enroll only patients with early or moderate-stage lymphedema. Traditionally, physiologic treatments of lymphedema, especially LVB, were reserved and thought to be most effective for patients with earlystage lymphedema, while patients with ISL stage III fibrofatty lymphedema were treated with debulking or excisional techniques, which aim to decrease the volume of the affected limb without any attempt at restoration of lymphatic flow. However, with improvements in imaging modalities and the increasingly frequent use of preoperative MRL and duplex ultrasound, functional lymphatics, located in the deep fat beneath the superficial fascia, can be detected. In their recent publication, Cha et al. [18] describe their success in using these imaging modalities to detect functional lymphatics that may not have been traditionally detected with ICG lymphography. With this approach, they were able to perform LVB on patients with stage 2-3 lymphedema, resulting in reduction in limb volume and subjective improvements in symptoms.
In addition to the feasibility of performing LVB on patients with advanced lymphedema, other studies have shown that VLNT in combination with debulking procedures, can also significantly impact and improve late-stage lymphedema of both the upper and lower extremities. Ciudad et al. [19] describe their technique of combining double gastroepiploic VLNTs with radical reduction with preservation of perforators. The 16 patients enrolled in their study, all had both improvements in limb volume reduction, as well as quality-of-life measurements. Similar improvements have also been shown when VLNT was combined with suction-assisted lipectomy [20].

A recent study from our institution also provides support for performing physiologic surgeries on patients with advanced lymphedema [5]. A chart review of a prospectively collected database of 274 patients with secondary upper and lower extremity lymphedema was performed, and follow-up volumetric and LLIS data up to 4 years postoperatively was analyzed. Similar to patients with ICG stage 1-2 lymphedema, patients with laterstage ICG 3-4 lymphedema showed a statistically significant reduction in both volume and LLIS scores postoperatively. Additionally, the results of a multivariate regression analysis showed that ICG stage was not associated with worse postoperative outcomes. While patients with earlier-stage lymphedema trended towards having slightly improved outcomes (although not statistically significant), the results from this study support the possible impact that physiologic surgery can have on patients with more advanced lymphedema.

\section{PRIMARY LYMPHEDEMA}

Although secondary lymphedema accounts for the majority of both upper and lower extremity lymphedema cases, primary lymphedema can often be a lifelong problem for those affected. The etiology of primary lymphedema, due to intrinsic problems with the lymphatics, differs from that of secondary lymphedema, where normal lymphatics are blocked, damaged, or disrupted. Because of this, many past studies examining the efficacy of physiologic surgical treatments of lymphedema have excluded these patients with primary lymphedema, and as such, the reported outcomes for procedures such as VLNT and LVB only applied to patients with secondary lymphedema. There is now increasing interest in whether VLNT and LVB play a role in improving symptoms associated with primary lymphedema. In their study of 17 patients with 19 lymphedematous limbs, Cheng et al. [21] performed 15 VLNT and 4 LVB, depending on whether functional lymphatics were present on ICG lymphography. They found that patients in both groups had significant volume reduction, improvement in quality of life, and fewer 
episodes of cellulitis postoperatively, although the VLNT patients tended to show more improvement compared to the LVB patients.

Our own experience treating patients with primary lymphedema corroborates these findings. In a group of 43 patients with primary lower extremity lymphedema, treated with VLNT, LVB, or both simultaneous VLNT and LVB, postoperative improvements in both volume reduction as well as quality-of-life measurements were seen, at both short-term and long-term followup (unpublished data). However, we found that these improvements, when compared to patients with secondary lower extremity lymphedema, were not as great. Thus, while physiologic surgical procedures show promise in the management of primary lymphedema, additional prospective studies are needed.

\section{LYMPHEDEMA PREVENTION}

While there are currently no cures for lymphedema, there has been much interest in immediate lymphatic reconstruction (ILR), also known as Lymphatic Microsurgical Preventive Healing Approach (LYMPHA), for the prevention of lymph- edema (Fig. 5) [22]. This technique, where LVB is performed at the time of axillary node dissection, was first described in 2009 by Boccardo et al. [23]. In their paper, they describe the outcomes of 19 patients who underwent this technique at the time of complex axillary node dissection, none of whom developed postoperative lymphedema at either the 6- or 12-month follow-up time points. More long-term results (4-year followup) have since been reported, showing a decreased rate of lymphedema compared to historical controls [24].

Since its initial description, there have been multiple descriptions of different techniques used for LYMPHA, as well as studies showing its efficacy in reducing lymphedema rates $[25,26]$. The success of LYMPHA in preventing lymphedema following axillary dissection was reported by Feldman et al. in 2015 [27]. In their study of 37 patients with a mean follow-up of 6 months, $50 \%$ who did not undergo LYMPHA developed secondary upper extremity lymphedema, compared to a lymphedema rate of $12.5 \%$ in patients who underwent LYMPHA. Interestingly, when the same group presented their long-term (minimum 4-year follow-up) data at the 2020 American Society of Reconstructive Microsurgery conference (Fort Lauderdale, FL, USA),
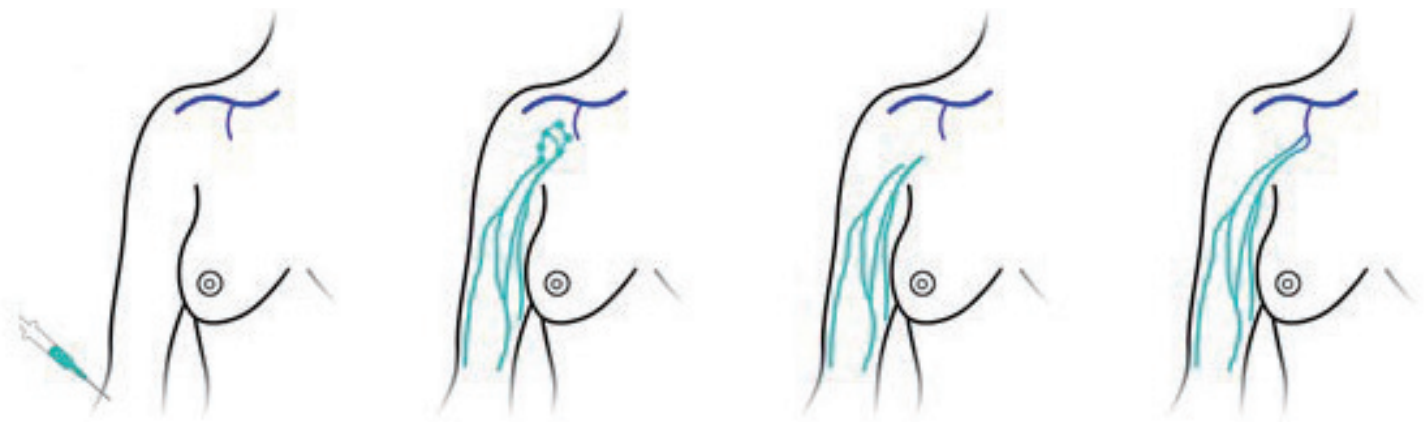

A

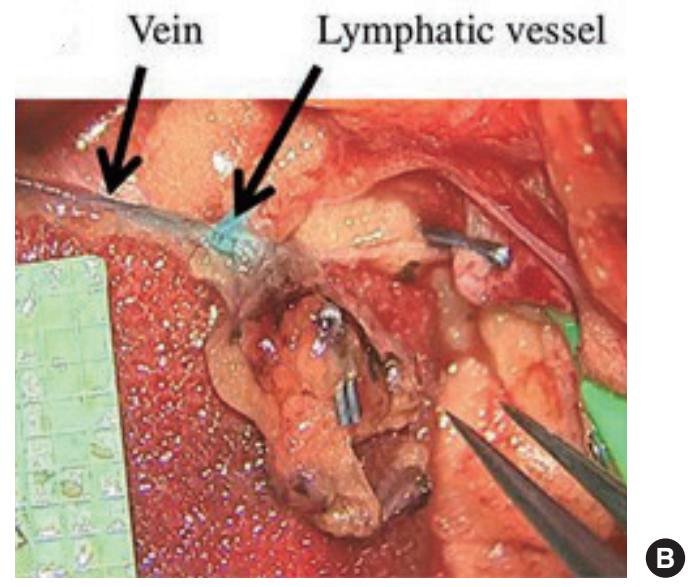

Fig. 5. Schematic and intraoperative photograph of Lymphatic Microsurgical Preventive Healing Approach (LYMPHA) technique. (A) Schematic demonstrating reverse lymphatic mapping with dye injection (green), detection of lymphatic vessels with dye (green), lymphadenectomy with removal of green lymph nodes, and microanastomoses between transected lymphatic vessels (green) and available vein branches (blue). (B) Intraoperative photograph showing microanastomoses between two lymphatic vessels and a single vein branch with evidence of patency with dye present in the vein branch. Reprinted from Agarwal et al. Breast J 2020;26:721-4, with permission from John Wiley and Sons [22]. 
they reported the incidence of lymphedema to be roughly the same between patients who underwent LYMPHA (31.3\% incidence) and those who did not (33.3\% incidence). From this data, they concluded that LYMPHA is not effective in reducing the risk of lymphedema long-term (data not yet published). A recent systematic meta-analysis by Jorgensen et al. [28] showed that treatment with prophylactic LVB reduced subsequent risk of developing lymphedema (relative risk 0.33), but also concluded that additional, high-quality studies on this topic must be performed in order to fully elucidate the impact of LYMPHA on preventing lymphedema.

In addition to the prevention of lymphedema at the time of axillary node dissection, the importance of early detection and intervention in cases of subclinical lymphedema is also increasingly being studied. In their retrospective cohort study of 1,790 patients, Bucci et al. [29] found that among patients undergoing either sentinel lymph node biopsy and axillary node dissection, the risk of breast cancer-related lymphedema was higher in those patients who experienced subclinical lymphedema, compared to those who did not. Given that the rate of lymphedema in patients undergoing complete axillary node dissection can be as high as $20 \%$ to $50 \%$, the ability to prevent even a portion of these cases at the time of initial surgery, as well as the increasing awareness and early interventions in patients with subclinical lymphedema, will have a large impact on decreasing the overall incidence of lymphedema.

In addition to its use for treatment of breast cancer-related lymphedema, prophylactic LVB has also been described in the treatment of lower extremity lymphedema following inguinal lymph node dissection for gynecologic or urologic malignancies, or in the treatment of extremity melanoma [30-32]. Similar to their results in patients who underwent LYMPHA of the upper extremity, Boccardo et al. [32] also showed that LYMPHA was effective in reducing lymphedema rates in 27 patients who underwent inguinofemoral lymphadenectomy for either vulvar cancer or melanoma of the trunk. Although these studies show somewhat promising results, usage of ILR/LYMPHA for prevention of lower extremity lymphedema, larger sample sizes and longer follow-up are required in order to make any definitive conclusions about use of this technique.

\section{NOTES}

\section{Conflict of interest}

David W. Chang is an editorial board member of the journal but was not involved in the peer reviewer selection, evaluation, or decision process of this article. No other potential conflicts of interest relevant to this article were reported.

\section{Author contribution}

Conceptualization: M Beederman, DW Chang. Data curation, formal analysis: M Beederman, DW Chang. Methodology, project administration: M Beederman, DW Chang. Visualization: M Beederman, DW Chang. Writing-original draft: M Beederman, DW Chang. Writing-review \& editing: M Beederman, DW Chang.

\section{ORCID}

Maureen Beederman https://orcid.org/0000-0001-7245-0949

David W. Chang https://orcid.org/0000-0002-8420-0098

\section{REFERENCES}

1. Rockson SG, Rivera KK. Estimating the population burden of lymphedema. Ann N Y Acad Sci 2008;1131:147-54.

2. Charles RH. The surgical treatment of elephantiasis. Ind Med Gaz 1901;36:84-99.

3. Chang DW, Masia J, Garza R 3rd, et al. Lymphedema: surgical and medical therapy. Plast Reconstr Surg 2016;138(3 Suppl):209S-218S.

4. Suami H, Chang DW. Overview of surgical treatments for breast cancer-related lymphedema. Plast Reconstr Surg 2010;126:1853-63.

5. Beederman M, Garza RM, Agarwal S, et al. Outcomes for physiologic microsurgical treatment of secondary lymphedema involving the extremity. Ann Surg 2020 Sep 1 [Epub]. https://doi.org/10.1097/SLA.0000000000004457.

6. Schaverien MV, Asaad M, Selber JC, et al. Outcomes of vascularized lymph node transplantation for treatment of lymphedema. J Am Coll Surg 2021;232:982-94.

7. Brazio PS, Nguyen DH. Combined liposuction and physiologic treatment achieves durable limb volume normalization in class II-III lymphedema: a treatment algorithm to optimize outcomes. Ann Plast Surg 2021;86(5S Suppl 3):S3849.

8. Chang DW, Dayan J, Greene AK, et al. Surgical treatment of lymphedema: a systematic review and meta-analysis of controlled trials: results of a consensus conference. Plast Reconstr Surg 2021;147:975-93.

9. Kwon JG, Hong DW, Suh HP, et al. Patient-specific surgical options for breast cancer-related lymphedema: technical tips. Arch Plast Surg 2021;48:246-53.

10. Schaverien MV, Coroneos CJ. Surgical treatment of lymphedema. Plast Reconstr Surg 2019;144:738-58.

11. Hayashi A, Yamamoto T, Yoshimatsu H, et al. Ultrasound visualization of the lymphatic vessels in the lower leg. Mi- 
crosurgery 2016;36:397-401.

12. Hayashi A, Hayashi N, Yoshimatsu H, et al. Effective and efficient lymphaticovenular anastomosis using preoperative ultrasound detection technique of lymphatic vessels in lower extremity lymphedema. J Surg Oncol 2018;117:290-8.

13. Hayashi A, Giacalone G, Yamamoto T, et al. Ultra high-frequency ultrasonographic imaging with $70 \mathrm{MHz}$ scanner for visualization of the lymphatic vessels. Plast Reconstr Surg Glob Open 2019;7:e2086.

14. Dayan JH, Wiser I, Verma R, et al. Regional patterns of fluid and fat accumulation in patients with lower extremity lymphedema using magnetic resonance angiography. Plast Reconstr Surg 2020;145:555-63.

15. Dayan JH, Dayan E, Kagen A, et al. The use of magnetic resonance angiography in vascularized groin lymph node transfer: an anatomic study.J Reconstr Microsurg 2014;30:41-5.

16. Yasunaga Y, Nakajima Y, Mimura S, et al. Magnetic resonance lymphography as three-dimensional navigation for lymphaticovenular anastomosis in patients with leg lymphedema. J Plast Reconstr Aesthet Surg 2021;74:1253-60.

17. Forte AJ, Boczar D, Huayllani MT, et al. Use of magnetic resonance imaging lymphangiography for preoperative planning in lymphedema surgery: a systematic review. Microsurgery 2021;41:384-90.

18. Cha HG, Oh TM, Cho MJ, et al. Changing the paradigm: lymphovenous anastomosis in advanced stage lower extremity lymphedema. Plast Reconstr Surg 2021;147:199207.

19. Ciudad P, Manrique OJ, Adabi K, et al. Combined double vascularized lymph node transfers and modified radical reduction with preservation of perforators for advanced stages of lymphedema.J Surg Oncol 2019;119:439-48.

20. Agko M, Ciudad P, Chen HC. Staged surgical treatment of extremity lymphedema with dual gastroepiploic vascularized lymph node transfers followed by suction-assisted lipectomy: a prospective study. J Surg Oncol 2018;117:114856.

21. Cheng MH, Loh CY, Lin CY. Outcomes of vascularized lymph node transfer and lymphovenous anastomosis for treatment of primary lymphedema. Plast Reconstr Surg Glob Open 2018;6:e2056.

22. Agarwal S, Garza RM, Chang DW. Lymphatic Microsurgi- cal Preventive Healing Approach (LYMPHA) for the prevention of secondary lymphedema. Breast J 2020;26:721-4.

23. Boccardo F, Casabona F, De Cian F, et al. Lymphedema microsurgical preventive healing approach: a new technique for primary prevention of arm lymphedema after mastectomy. Ann Surg Oncol 2009;16:703-8.

24. Boccardo F, Casabona F, De Cian F, et al. Lymphatic microsurgical preventing healing approach (LYMPHA) for primary surgical prevention of breast cancer-related lymphedema: over 4 years follow-up. Microsurgery 2014;34:421-4.

25. Coriddi M, Mehrara B, Skoracki R, et al. Immediate lymphatic reconstruction: technical points and literature review. Plast Reconstr Surg Glob Open 2021;9:e3431.

26. Johnson AR, Fleishman A, Granoff MD, et al. Evaluating the impact of immediate lymphatic reconstruction for the surgical prevention of lymphedema. Plast Reconstr Surg 2021;147:373e-381e.

27. Feldman S, Bansil H, Ascherman J, et al. Single institution experience with Lymphatic Microsurgical Preventive Healing Approach (LYMPHA) for the primary prevention of lymphedema. Ann Surg Oncol 2015;22:3296-301.

28. Jorgensen MG, Toyserkani NM, Sorensen JA. The effect of prophylactic lymphovenous anastomosis and shunts for preventing cancer-related lymphedema: a systematic review and meta-analysis. Microsurgery 2018;38:576-85.

29. Bucci LK, Brunelle CL, Bernstein MC, et al. Subclinical lymphedema after treatment for breast cancer: risk of progression and considerations for early intervention. Ann Surg Oncol 2021 Jun 11 [Epub]. https://doi.org/10.1245/s10434021-10173-0.

30. Morotti M, Menada MV, Boccardo F, et al. Lymphedema microsurgical preventive healing approach for primary prevention of lower limb lymphedema after inguinofemoral lymphadenectomy for vulvar cancer. Int J Gynecol Cancer 2013;23:769-74.

31. Cakmakoglu C, Kwiecien GJ, Schwarz GS, et al. Lymphaticovenous bypass for immediate lymphatic reconstruction in locoregional advanced melanoma patients. J Reconstr Microsurg 2020;36:247-52.

32. Boccardo F, Valenzano M, Costantini S, et al. LYMPHA technique to prevent secondary lower limb lymphedema. Ann Surg Oncol 2016;23:3558-63. 